\title{
The Foam Analogy in Charged Colloidal Crystals
}

\author{
William Kung, P. Ziherl, f and Randall D. Kamien \\ Department of Physics and Astronomy, University of Pennsylvania, Philadelphia, PA 19104-6396
}

(Dated: November 3, 2018)

\begin{abstract}
We model charged colloidal suspensions using an analogy with foams. We study the solid-solid phase transitions of these systems as a function of particle volume-fraction and ionic strength. The screened Coulomb interaction is replaced by an interaction between walls of the Voronoi cells around each particle. We fit the surface charge to reproduce the phase diagram for the charged suspension studied by Sirota et al. [Phys. Rev. Lett. 62, 1524 (1989)]. With this fit parameter we are able to calculate the elastic moduli of the system and find good agreement with the available data.
\end{abstract}

PACS numbers: 61.50.Ah, 62.20.Dc

Charged colloids have been the subject of intense study both experimentally and theoretically. In the laboratory, optical techniques can readily probe these systems and they are easily manipulated via chemical means 11, 2, 3, 4. 1. Their rich chemistry leads to many industrial applications ranging from uses in coating materials, in ceramic precursors, and in designing and manufacturing biological macromolecules. These systems interact through a short-range screened Coulomb potential and thus epitomize one of the few classes of interactions in soft systems. In this Letter, we replace the exact screened-Coulomb interaction between colloidal spheres with an effective interaction proportional to the surface area of the Wigner-Seitz or Voronoi cells which contain each sphere. By balancing the entropic interaction which favors the close-packed face-centered cubic (FCC) lattice with the surface interaction which, as we will argue, favors the body-centered cubic (BCC) lattice, we will find the phase coexistence line as a function of volume fraction and salt concentration. We compare our results to experiment to find the surface charge on the spheres. Using this fitted value, we calculated the shear moduli within our framework and found close agreement with experiment. Our model leads to quantitatively different results than those found in molecular dynamics (MD) simulations [5] of particles interacting via Yukawa interactions.

Several experimental studies [1, 2, 3] provide a wealth of data on the stability of colloidal phases, in particular, the fluid phase, the glass phase and two solid phases with either FCC or BCC lattices. These systems are aqueous suspensions of uniformly charged polystyrene spheres with a variable salt concentration which is a control parameter for the degree of screening of the underlying Coulomb interaction. Because the lattice spacing is large in the ordered phases, they can be probed by scattering of visible light [- 1 . Through scattering it is possible to determine those volume fractions at which the orderdisorder transition occurs at low salt concentrations and

*Permanent address: J. Stefan Institute, Jamova 39, SI-1000 Ljubljana, Slovenia

${ }^{\dagger}$ Electronic address: kamien@physics.upenn.edu at which the FCC-BCC transition occurs. Though the MD simulations [5] qualitatively corroborate these experimental findings, one is hard-pressed for similar quantitative agreement between existing experimental data and simulation results. The lack of exact analytic methods with which to compare experiment or simulation further complicates this problem. Thus theoretical models which distill the crucial aspects of the interactions and lead to testable predictions are necessary.

Recently, a foam analogy was used to account for the many non-closed packed structures observed in colloidal and lyotropic systems [6, 7]. In this analogy, the surface interaction replaced the brush-like interaction between the spheres and accounted for the entropic degrees of freedom associated with configurations of the soft coronas of alkyl chains attached to hard cores. Since the coronas prefer a packing arrangement that disfavors interdigitation and thus maximize the average separation of the cores, lattices with small interfacial area are favored in such systems. For example, the BCC lattice of orthic tetrakaidecahedra has a smaller area than the FCC lattice of rhombic dodecahedra, and so the surface interaction favors the BCC lattice.

Here we apply the same framework to understanding the experimental phase diagram 8 and MD simulation results [5 for charged colloidal systems. In particular, our model couples the bulk free energy of the charged hard cores to the free energy of their screened Coulomb interaction via the effective surface area. The model contains a single free parameter: the surface potential associated with each colloidal particle. Using experiment as our guide, we fit the surface potential to reproduce the phase diagram. In order to test our theory, we use the same surface potential to calculate the shear and bulk moduli and compare these with experiment and simulation. We have found good agreement with the available data and, in doing so, demonstrate the plausibility of understanding the various experimental results of charged systems in terms of a geometrical principle already established for another class of colloids.

An apparent dramatic feature of our results is the negative values of the bulk modulus for small $\lambda=\kappa a$, where $\kappa$ is the inverse Debye screening length and $a$ is the average interparticle spacing. This is an artifact of our 
model which can be simply understood: for $\lambda=1$ the Debye screening length is exactly equal to the interparticle spacing. As a result, we would expect next-to-nearestneighbor interactions to play an important role in the stability and energetics of the charged colloidal crystal and therefore would not trust our model at small $\lambda$. However, for larger values of $\lambda$ the bulk modulus become positive - for the data we compare with, $\lambda \approx 4.64$, well within the regime where next-to-nearest-neighbor interactions are small. Our model also yields qualitatively different behavior for the relative magnitudes of the elastic constants in comparison to MD simulations [5. We attribute this difference to the finite size of the colloids - in the MD simulation they were treated as point particles and thus did not suffer entropic losses from excluded volume. We also note that the shear modulus becomes negative at large values of $\lambda$. This signals the instability of the BCC lattice and its transition to FCC: for large $\lambda$ the screenedCoulomb potential becomes unimportant and the system should behave as if the interactions were purely hardcore. This is in line with the recent experimental determination [9] that the FCC lattice is the equilibrium phase for samples with $\lambda>10$. Moreover, in that study it was argued that since Yukawa-like pair interactions could not account for both the elasticity of the crystal and the colloidal dynamics, three-body and higher interactions must play an important role. Since our approach is intrinsically many-body, our results for the elastic constants as a function of $\lambda$ are consistent with that conclusion - we find behavior that cannot be explained by MD simulation of particles interacting via Yukawa potentials. There have been alternative explanations of this discrepancy based on hydrodynamic screening $[10$. We hope that our framework will allow for improved understanding of these issues.

In a colloidal suspension at fixed density, the volume of the container is the sum of the volume of the hard spheres and the excess volume of the salt solution. Since this latter volume can be viewed as enveloping the individual spheres, we can imagine breaking the volume up into a lattice of Voronoi cells, each of which contains a colloidal particle. The excess volume can then be written as the product of the area of these dividing surfaces and their average thickness so that

$$
A d=\text { constant }
$$

where $A$ is total area of these bilayers and $d$ is their average thickness. Maximizing $d$ to reduce the repulsive interaction amounts to minimizing $A$ whence comes the interaction that favors BCC lattices (and the more exotic A15 lattices [6, 11]). Since the crystal lattice is composed of identical colloidal particles in cases of interest, the minimum area problem is equivalent to Kelvin's problem [12] of partitioning a given space into cells of equal volumes having the smallest interfacial area.

To compute the bulk free energy of the system, we follow the method developed in a previous study [6, 7] by adopting the cellular free-volume theory. In this approx- imation, each particle is confined to a cage formed by its neighbors. The free volume available to each particle's center of mass is the volume of the Wigner-Seitz cell after a layer of thickness $\sigma / 2$ (where $\sigma$ is the hard-core diameter of particles) is peeled off of its faces. Despite it mean-field nature, the free volume theory yields excellent quantitative agreement with available numerical simulations in the high-density limit and is roughly $1 \%$ off above the hard-sphere fluid-solid transition. The use of free volume theory at the lower densities probed in the charged colloidal systems could be suspect. However as long as the shear elastic constants are non-zero, we expect that an "Einstein-crystal" description of the phonon modes should be adequate. Indeed, we will find that appropriate moduli are non-zero in the density and salt concentration regime we are studying [13] and therefore there are no "soft modes" which might strongly contribute to collective effects. The resulting bulk free energy for the FCC or BCC lattice is:

$$
F_{\text {bulk }}^{X}=-k_{B} T \ln \left(\alpha^{X}\left(\beta^{x} n^{-1 / 3}-1\right)^{3}\right),
$$

where $n=\rho \sigma^{3}$ is the reduced density and $\sigma$ is the hardcore diameter of each colloidal particle. The coefficients $\alpha^{F C C}=2^{5 / 2}$ and $\alpha^{B C C}=6.716$ depend on the shape of the cells, whereas $\beta^{F C C}=2^{1 / 6}$ and $\beta^{B C C}=2^{-2 / 3} 3^{1 / 2}$ are determined by their size.

To obtain the electrostatic energy between the charged spheres, we model their interaction as that between two charged flat plates using the Debye-Hückel approximation 14 for a salt solution with counterions of charge $Z e$. We replace each sphere with its Voronoi cell and then, in a Derjaguin-like approximation, replace the interaction with an interaction across parallel plates. The energy between two plates can be found via an electromagnetic potential satisfying the linearized Poisson-Boltzmann equation. For the experimental system in question we will show that this approximation is valid. Using linear superposition and the solution for a single charged plate, we have [3]:

$$
F_{\mathrm{c}}=64 A_{\mathrm{M}} k_{B} T n_{\mathrm{b}} \kappa^{-1} \tanh ^{2}\left(\frac{1}{4} \Psi_{\mathrm{s}}\right) \exp (-\kappa d) .
$$

Taken together, Eqs. (2) and (3) encode the complete picture of the structural FCC-BCC transition in terms of the bulk counterion number density $n_{\mathrm{b}}$, the dimensionless surface potential of colloids $\Psi_{\mathrm{s}}$, the colloid density $n$, and the Debye screening length $\kappa^{-1}=\sqrt{\epsilon k_{B} T / 2 e^{2} Z^{2} n_{b}}$, itself a function of these control variables (where $\epsilon$ is the dielectric constant). In order to relate the interaction energy $F_{\mathrm{c}}$ to the reduced density $n$, we relate the spacing of the charged plates to the density via the constraint Eq. (11). The interparticle separation $d$ depends on the particle density:

$$
A_{\mathrm{M}} d=2\left(\frac{1}{n}-\frac{\pi}{6}\right) \sigma^{3},
$$


where $A_{\mathrm{M}}=\gamma^{X} \sigma^{2} n^{-2 / 3}$, and $\gamma^{F C C}=5.345$ and $\gamma^{B C C}=5.308$ are dimensionless quantities characterizing the magnitude of area. Substitution of Eq. (4) into Eq. (3) completes the expression for the interfacial free energy. Since the density and salt concentration are measurable parameters, the only free parameter is the surface potential $\Psi_{\mathrm{s}}$. It is therefore customary to treat $\Psi_{\mathrm{s}}$ as a parameter chosen for the best fit to the data 114. From the surface potential we will be able to determine the net surface charge $q$ on the colloidal spheres.

Focusing on the structural phase transition from BCC to FCC, we rely on the X-ray scattering study of charged polystyrene spheres by Sirota et al. [8]. That experiment studied the phase diagram of polystyrene spheres in a 0.9-methanol-0.1-water suspension. The thermodynamic behavior of this system was recorded as a function of volume fraction $\phi$ and salt concentration $c_{\mathrm{HCl}}$. In order to determine the surface potential, we choose an FCC$\mathrm{BCC}$ coexistence point in the experimental data, and then equate the corresponding FCC and BCC free energies to solve for $\Psi_{\mathrm{s}}$. Through Gauss's law, we calculate the charge per colloid $q=2 A_{M} \sqrt{2 \epsilon k_{B} T n_{b}} \sinh \left(\frac{1}{2} \Psi_{\mathrm{s}}\right)$. Finally, using this value of the charge, we find the density $n$ at which $F^{F C C} / k_{B} T$ and $F^{B C C} / k_{B} T$ are equal for each salt concentration $n_{\mathrm{b}}$. Due to the limited data, there is only one experimental point at non-zero salt concentration that is in the coexistence region. We thus obtain the coexistence curve in $\left(\phi-n_{\mathrm{b}}\right)$ phase diagram (Fig. 11). The relevant experimental data from Ref. [\$] are included for comparison.

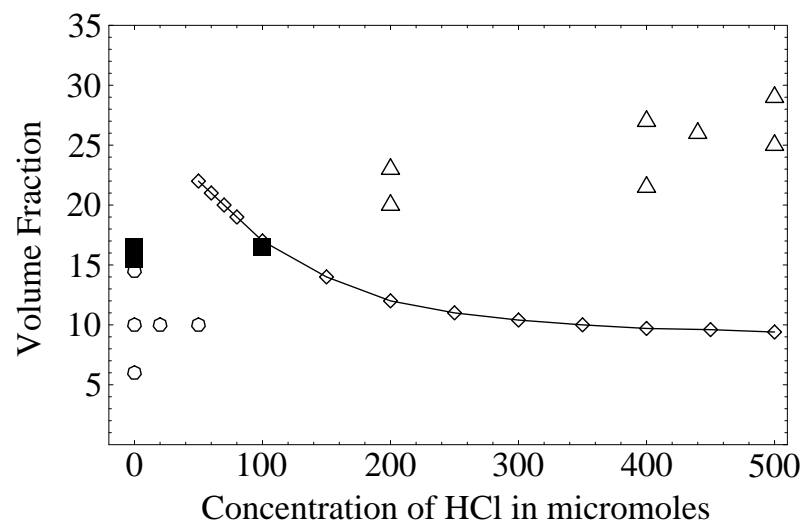

FIG. 1: Theoretical FCC-BCC coexistence curve as a function of volume fraction $\phi$ and electrolyte concentration $(\mathrm{HCl})$. The diamonds are the theoretical predictions, while the other points come from the data of Ref. [8]. Solid squares are coexistence points, open triangles are FCC, and open circles are BCC.

Within our framework we find a surface potential of $\Psi_{\mathrm{s}}=0.2$, and so we were justified in linearizing the Poisson-Boltzmann equation. However, this potential corresponds to a total charge of about $48 e$ per colloidal particle. The value of the charge is smaller than the quoted experimental value of about 135 e per sphere [8], though it is comparable in magnitude. The experimental value was actually determined indirectly by measuring the shear modulus for the crystalline sample at $12 \%$ volume fraction [15]. Because of the uncertainty in the charge, we will make a direct comparison with experiment by calculating the shear modulus of the BCC lattice using our cellular framework.

In general, the elastic energy of a cubic crystal has three elastic constants, $K_{11}, K_{12}$, and $K_{44}$ [16]:

$$
\begin{array}{r}
F_{\text {cubic }}=\frac{1}{2} \int \mathrm{d}^{3} x\left[K_{11}\left(u_{x x}^{2}+u_{y y}^{2}+u_{z z}^{2}\right)\right. \\
+K_{12}\left(u_{x x} u_{y y}+u_{x x} u_{z z}+u_{y y} u_{z z}\right) \\
\left.+2 K_{44}\left(u_{x y}^{2}+u_{x z}^{2}+u_{y z}^{2}\right)\right] .
\end{array}
$$

For a polycrystalline sample, the bulk modulus $K=$ $\left(K_{11}+2 K_{12}\right) / 3$ is an isotropic quantity. On the other hand, the shear modulus depends on the direction of the applied shear and ranges from the elongational shear $\mu=K_{11}-K_{12} / 2$ to the simple shear $K_{44}$. We determine the three elastic constants by calculating the free volume and the change in surface area for a deformed BCC lattice. The free volume was found via a simple numerical scheme while the change in surface area was found with the aid of Surface Evolver [17]. Equating the continuum energy change from Eq. (5) with the energy change within our framework, we find that $K_{11} \approx 17.1 \mathrm{~N} / \mathrm{m}^{2}$, $K_{12} \approx-1.2 \mathrm{~N} / \mathrm{m}^{3}$, and $K_{44} \approx 10.3 \mathrm{~N} / \mathrm{m}^{2}$ for a $12 \%$ volume-fraction $(n=0.23)$ BCC sample. Thus the shear modulus ranges from 10.3 to $17.7 \mathrm{~N} / \mathrm{m}^{2}$. Again this is the same order of magnitude as the measured, isotropic value of $17 \mathrm{~N} / \mathrm{m}^{2}$, which is a complicated combination of the two shear moduli. We have calculated these moduli using an $\mathrm{HCl}$ concentration of $50 \mu \mathrm{mol}$, as this is in the middle of the reported BCC regime [8].

In order to compare with MD simulations [5], we tabulate (Table II) values of the bulk modulus and the three elastic constants as a function of $\lambda=\kappa a$ (in the experimental study, $\lambda \approx 4.64$ ). However, unlike point particles with Yukawa interactions, our model does not scale simply with this parameter. To vary $\lambda$, we keep the colloidal density fixed as well as the total charge on each colloid. Varying the salt concentration changes both $\kappa$ and the surface potential $\Psi_{\mathrm{s}}$. With these new values we can recalculate the elastic constants. For completeness, we tabulate the values of the bulk modulus $K$ and elastic constants of the BCC structure at $\phi=0.12$ for different values of $\lambda=\kappa a$ (where $a$ is the mean separation of spheres; at $n=0.23, a \approx 140 \mathrm{~nm})$ :

While our framework assigns a surface charge that is smaller than the value suggested in the original analysis [8], it is in fact quite common for the effective charge to be reduced when compared with the net or titratable charge. To calculate the renormalized charge $Z^{*}$ and acid concentration $n_{\mathrm{HCl}}^{*}$, Alexander et al. [18] employed a Wigner-Seitz approximation similar in principle to our way of determining the bulk free energies of the various 


\begin{tabular}{ccccc}
$\lambda$ & $K$ & $K_{11}$ & $K_{12}$ & $K_{44}$ \\
\hline 1 & $-26.1 /-7.59$ & $17.5 / 10.7$ & $-47.9 /-16.7$ & $54.4 / 28.4$ \\
2 & $-7.54 / 13.8$ & $19.0 / 18.4$ & $-21.1 / 11.6$ & $29.1 / 15.6$ \\
3 & $0.12 / 22.3$ & $19.7 / 21.4$ & $-9.67 / 22.7$ & $18.3 / 10.6$ \\
4 & $3.66 / 26.2$ & $18.4 / 22.4$ & $-3.71 / 28.0$ & $12.7 / 7.95$ \\
5 & $5.37 / 28.0$ & $16.6 / 22.5$ & $-0.22 / 30.7$ & $9.40 / 6.40$ \\
6 & $6.16 / 28.6$ & $14.5 / 22.0$ & $2.00 / 32.0$ & $7.30 / 5.43$ \\
7 & $6.46 / 28.7$ & $12.5 / 21.1$ & $3.40 / 32.5$ & $5.90 / 4.70$ \\
8 & $6.49 / 28.5$ & $10.6 / 20.3$ & $4.42 / 32.6$ & $5.00 / 4.30$
\end{tabular}

TABLE I: Calculated values for the bulk modulus $K$ and elastic constants of the BCC structure at several values of $\lambda=\kappa a$, where $a$ is the average interparticle spacing, for $n=0.23$ (first number) and $n=0.50$ (second number). All values are in units of $\mathrm{N} / \mathrm{m}^{2}$.

lattices. Their method relies on the fact that in interstitial regions, which occupy a large fraction of the system, the potential $\Psi$ is relatively uniform and changes abruptly in a boundary layer near the colloidal surfaces. As a result, the counterions are more tightly constrained to locations near the surfaces of these colloidal particles, and one can re-interpret these ions as part of an effective sphere with a reduced charge. Again, since neither the effective charge nor the local ion concentration can be measured directly in experiments, the precise confirmation of theory has proven difficult. Because our model allows the simultaneous prediction of the phase boundary and the shear moduli, we can avoid these uncertainties.

There are, of course, additional interactions that we have neglected. The van der Waals attraction is much weaker than the screened Coulomb potential at the interparticle spacing of $a \approx 140 \mathrm{~nm}$. We expect that correlation effects such as overcharging should have minimal consequence in a system of monovalent salt ions of
$\mathrm{NaCl}$ 19]. Dispersion forces are important at ion concentrations greater than $100 \mu \mathrm{mol}$ [20]. However, this effect occurs at distance scales on the order of $10 \mu \mathrm{m}$, and we do not expect this to change our model.

Finally, we note that in the calculation of the bulk free energy, we employ free-volume theory even in the relatively low-particle-density regime - a regime in which pure hard-core interactions would predict a fluid phase. However, the screened Coulomb potential stabilizes the lattice structures and, in turn, lowers the melting density of the system. One could characterize the system with an effective density of higher magnitude, determined by the Barker-Henderson effective diameter of particles [14]. A scheme like this would introduce another parameter into our model which would divide the screened-Coulomb potential into a "hard" part and a "soft" part.

We have established a geometrical framework for understanding the structural and mechanical properties of charged colloids. We have connected the phase diagram to the elastic moduli of the system with only one adjustable parameter, the surface charge. The balance between the drive of the system's entropy for maximal packing fraction and the need of its screened repulsion for minimal interfacial area accounts for most of the properties found in experiments. Its elucidation can serve as an intuitive guide to the engineering of all such charged systems.

We gratefully acknowledge stimulating conversations with T.C. Lubensky, T.R. Powers, M.O. Robbins, D.A. Weitz, and A.G. Yodh. This work was supported by NSF Grant DMR97-32963, the Donors of the Petroleum Research Fund, administered by the American Chemical Society, the University of Pennsylvania Research Foundation, and a gift from L.J. Bernstein. R.D.K. was also supported by the Alfred P. Sloan foundation.
[1] S. Hachisu, Y. Kobayashi, and A. Rose, J. Col. Int. Sci. 42, 342 (1973).

[2] T. Yoshiyama, Polymer 27, 828 (1986).

[3] W. B. Russel, D. A. Saville, and W. R. Schowalter, Colloidal Dispersions (Cambridge University Press, New York, 1989).

[4] Y. Monovoukas and A. P. Gast, J. Col. Int. Sci. 128, 533 (1989).

[5] M. O. Robbins, K. Kremer, and G. S. Grest, J. Chem. Phys. 88,3286 (1988).

[6] P. Ziherl and R. D. Kamien, Phys. Rev. Lett. 85, 3528 (2000).

[7] P. Ziherl and R. D. Kamien, J. Phys. Chem. B 105, 10147 (2001).

[8] E. B. Sirota, et al., Phys. Rev. Lett. 62, 1524 (1989).

[9] J. A. Weiss, A. E. Larsen, and D. G. Grier, J. Chem. Phys. 109, 8659 (1998).

[10] T. M. Squires and M. P. Brenner, Phys. Rev. Lett. 85, 4976 (2000).
[11] V. S. K. Balagurusamy, G. Ungar, V. Percec, and G. Johansson, J. Am. Chem. Soc. 119, 1539 (1997).

[12] W. Thomson, Phil. Mag. 24, 503 (1887).

[13] We thank M. O. Robbins for discussions on this point.

[14] A. P. Gast, C. K. Hall, and W. B. Russel, Faraday Discuss. Chem. Soc. 6, 189 (1983).

[15] J. F. Joanny, J. Col. Int. Sci. 71, 622 (1979).

[16] P. M. Chaikin and T. C. Lubensky, Principles of Condensed Matter Physics (Cambridge University Press, New York, 1980).

[17] K. Brakke, Exp. Math. 1, 141 (1992).

[18] S. Alexander, P. M. Chaikin, P. Grant, G. J. Morales, P. Pincus, and D. Hone, J. Chem. Phys. 80, 5776 (1984).

[19] T. T. Nguyen, A. Yu. Grosberg, and B. I. Shklovskii, Phys. Rev. Lett. 85, 1568 (2000).

[20] M. Boström, D. R. M. Williams, and B. W. Ninham, Phys. Rev. Lett. 87, 168103 (2001). 